\title{
Discrete Abelian Gauge Theories for Quantum Simulations of QED
}

\author{
Simone Notarnicola ${ }^{1}$, Elisa Ercolessi ${ }^{2,3}$, Paolo Facchi ${ }^{4,5}$, \\ Giuseppe Marmo ${ }^{6,7}$, Saverio Pascazio ${ }^{4,5}$, Francesco V. Pepe Pe,5, $^{4,5}$ \\ ${ }^{1}$ SISSA, International School for Advanced Studies, via Bonomea 265, I-34136 \\ Trieste, Italy \\ ${ }^{2}$ Dipartimento di Fisica e Astronomia dell'Università di Bologna, Via Irnerio 46, \\ I-40127 Bologna, Italy \\ ${ }^{3}$ INFN, Sezione di Bologna, Via Irnerio 46, I-40127 Bologna, Italy \\ ${ }^{4}$ Dipartimento di Fisica and MECENAS, Università di Bari, I-70126 Bari, Italy \\ ${ }^{5}$ INFN, Sezione di Bari, I-70126 Bari, Italy \\ ${ }^{6}$ Dipartimento di Fisica and MECENAS, Università di Napoli "Federico II", I-80126 \\ Napoli, Italy \\ ${ }^{7}$ INFN, Sezione di Napoli, I-80126 Napoli, Italy \\ ${ }^{8}$ Museo Storico della Fisica e Centro Studi e Ricerche "Enrico Fermi", I-00184 Roma, \\ Italy
}

\begin{abstract}
We study a lattice gauge theory in Wilson's Hamiltonian formalism. In view of the realization of a quantum simulator for QED in one dimension, we introduce an Abelian model with a discrete gauge symmetry $\mathbb{Z}_{n}$, approximating the $U(1)$ theory for large $n$. We analyze the role of the finiteness of the gauge fields and the properties of physical states, that satisfy a generalized Gauss's law. We finally discuss a possible implementation strategy, that involves an effective dynamics in physical space.

PACS numbers: 37.10.Jk, 11.15.Ha, 67.85.-d, 42.50.Ex
\end{abstract}

Keywords: Quantum simulators, ultracold gases, lattice gauge theories.

\section{Introduction}

Recent development in low-temperature physics and atomic control techniques is providing the basic tools for setting up quantum simulators [1, 2, 3, 4]. The experimental feasibility of a quantum simulator will open the way to a more comprehensive understanding of complex systems and fundamental physics. An appealing application is the simulation of lattice gauge theories [5, 6, 7, 8, 9, 10]. The discretization of high-energy physics theories on lattices was initially motivated by the possibility to simulate them by classical computation. However, the complex nature of gauge theories represents a severe obstruction, which can be overcome through a quantum simulation. 
Atoms on a lattice [11, 12, 13, 14, 15, 16, 17, 18, 19, 20, 21] provide a natural toolbox to perform this task.

In this article we construct a lattice model which simulates an Abelian gauge theory. The model will be characterized by the interaction between an Abelian gauge (electromagnetic) field and a fermionic matter field, as in quantum electrodynamics (QED). We shall restrict our analysis to the one-dimensional case. As in the consolidated quantum link model (QLM) [22, 23, 24, 25], our system will be an approximation to QED in which the electric field can take a finite number of values. We will eventually discuss the possibility to implement the model on a cold atomic simulator. This task involves first the identification of the degrees of freedom of the simulator with those of the model, and then the correct implementation of the dynamics.

\section{Lattice QED}

The dynamics of the continuum-space QED in $1+1$ dimensions (Schwinger model) has its lattice counterpart in the Hamiltonian [6, 25, 26]

$$
H=-t \sum_{x}\left(\psi_{x}^{\dagger} U_{x, x+1} \psi_{x+1}+\text { H.c. }\right)+m \sum_{x}(-1)^{x} \psi_{x}^{\dagger} \psi_{x}+\frac{g^{2}}{2} \sum_{x} E_{x, x+1}^{2},
$$

with $x$ labelling the sites of a one-dimensional lattice. Fermionic matter is represented by the one-component spinor field operators $\psi_{x}$, defined on each site, which obey the canonical anticommutation relations $\left\{\psi_{x}, \psi_{x^{\prime}}^{\dagger}\right\}=\delta_{x, x^{\prime}},\left\{\psi_{x}, \psi_{x^{\prime}}\right\}=0$. The parameter $m$ is the fermion mass, while the staggered fermions with parity factor $(-1)^{x}$ are introduced in order to avoid the fermion-doubling problem in the discretization of the theory [8, 9, 5, 6]: the positive and negative-energy components of the Dirac spinor are encoded respectively in the even and odd lattice sites. In a simplified model, spinless particles are considered, with the spinor $\psi_{x}$ reducing to a single-component field. The gauge fields are instead defined on the links $(x, x+1)$ of the lattice. In the canonical gauge, which is the most convenient choice to develop a lattice gauge theory, the electric field $E$ and the vector potential $A$ are conjugated variables with canonical Commutation Relations (CR) $\left[E_{x, x+1}, A_{x^{\prime}, x^{\prime}+1}\right]=\mathrm{i} \delta_{x, x^{\prime}}$ [27]. Since the unitary operators $U$ (comparators [28]) in the hopping terms of (1) are locally related to the vector potential by exponentiation, $U_{x, x+1}=\mathrm{e}^{-\mathrm{i} A_{x, x+1}}$, they satisfy

$$
\left[E_{x, x+1}, U_{x^{\prime}, x^{\prime}+1}\right]=\delta_{x, x^{\prime}} U_{x, x+1} .
$$

The presence of the free electric field energy, with coupling constant $g^{2}$, thus yields a nontrivial dynamics for the comparators. Notice the absence of magnetic contributions to the Hamiltonian, a consequence of the one-dimensional nature of the system.

The terms $\psi_{x}^{\dagger} U_{x, x+1} \psi_{x+1}$ in (1) describe site hopping of fermions, related to a shift in the electric field. These contributions come from the discretization and integration on the lattice cells of the minimal-coupling terms $-\mathrm{i} \psi(x)^{\dagger} \gamma_{0} \gamma^{j} D_{j} \psi(x)$, with $\gamma^{\mu}$ the Dirac matrices, and $D_{j}=\partial_{j}+i A_{j}(x)$ the covariant derivatives [8, 28]. The minimal coupling ensures the symmetry of the Hamiltonian under local $U(1)$ transformations. 
Given a real function on the lattice, $\alpha_{x}$, local phase transformations $\psi_{x} \rightarrow \psi_{x} \mathrm{e}^{\mathrm{i} \alpha_{x}}$ and $U_{x, x+1} \rightarrow \mathrm{e}^{\mathrm{i} \alpha_{x}} U_{x, x+1} \mathrm{e}^{-\mathrm{i} \alpha_{x+1}}$ of the field operators leave the Hamiltonian (1) invariant. Due to the (anti)commutation properties of the fields, the phase transformation on any operator $F$ can be implemented through the application of

$$
\begin{aligned}
F & \rightarrow \prod_{x} \mathrm{e}^{-\mathrm{i} \alpha_{x} G_{x}} F \prod_{y} \mathrm{e}^{\mathrm{i} \alpha_{y} G_{y}}, \\
G_{x} & =\psi_{x}^{\dagger} \psi_{x}+\frac{1}{2}\left[(-1)^{x}-1\right]-\left(E_{x, x+1}-E_{x-1, x}\right) .
\end{aligned}
$$

The gauge transformation acts trivially on the local- $U(1)$ invariant Hamiltonian (1). For a generic operator $F$, the invariance property is equivalent to the $\mathrm{CR}\left[F, G_{x}\right]=0$ for all sites. The choice of the canonical gauge has the disadvantage that Gauss's law cannot be implemented at the operator level [27, 25]. Thus, not all the states in the total Hilbert space $\mathcal{H}$ of the system are physically acceptable. The invariance condition of a state $|\phi\rangle$ under any gauge transformation $\prod_{x} \mathrm{e}^{-\mathrm{i} \alpha_{x} G_{x}}|\phi\rangle=|\phi\rangle$ selects the Hilbert subspace

$$
\mathcal{H}_{G}=\left\{|\phi\rangle \in \mathcal{H}, G_{x}|\phi\rangle=0 \text { for all sites } x\right\},
$$

where, as can be deduced from (3), the charge $\psi_{x}^{\dagger} \psi_{x}$ and the divergence of the electric field $E_{x, x+1}-E_{x-1, x}$ are correctly related. The term multiple of the identity appearing in $G_{x}$ ensures that the vacuum state with vanishing electric field and all the negative-mass sites occupied (the Dirac sea) is in the gauge-invariant subspace $\mathcal{H}_{G}$.

\section{Finite link spaces: a $\mathbb{Z}_{n}$ model.}

In Wilson's original formulation of lattice gauge theories [7], the gauge operators on links act on infinite-dimensional Hilbert spaces, and both the electric field and the vector potential have continuum and unbounded spectra. A problem arises in quantum simulators, when one has to match the (infinite dimensional) link with an experimentally feasible and controllable system, with a finite number of levels. Two possible approaches are possible. The first one preserves for all dimensions the structure of the Hamiltonian (1), including the coupling of the matter fields with a unitary gauge operator. The second approach, which has been followed in the formulation of the QLM [22], consists in preserving the CR between field operators, (2) in particular. Since in quantum mechanics one is used to think in terms of commutators, the latter approach has so far appeared more natural. Unfortunately, this procedure focuses on the invariance with respect to the $\mathrm{U}(1)$ group of local transformations, at the expenses of the structure of the hopping term, that no longer involves a unitary comparator (minimal coupling prescription). We shall rather insist on the unitarity of the comparator thereby obtaining a bona fide lattice gauge theory for any dimension of the link Hilbert space. Let us first observe that a gauge transformation (3) acts on the comparator $U_{x, x+1}=\mathrm{e}^{-\mathrm{i} A_{x, x+1}}$ as

$$
U_{x, x+1} \rightarrow \mathrm{e}^{\mathrm{i}\left(\alpha_{x}-\alpha_{x+1}\right) E_{x, x+1}} U_{x, x+1} \mathrm{e}^{-\mathrm{i}\left(\alpha_{x}-\alpha_{x+1}\right) E_{x, x+1}}=\mathrm{e}^{\mathrm{i}\left(\alpha_{x}-\alpha_{x+1}\right)} U_{x, x+1} .
$$


This result is indeed a special case of a general property of operators in the Weyl group generated by the conjugated operators $A$ and $E$ on a link (indices will be omitted for clarity) [29, 30]. Indeed, the electric field and the vector potential are the generators of the two-parameter projective unitary (Weyl) group $\left\{\mathrm{e}^{\mathrm{i}(\xi E-\eta A)}\right\}_{\xi, \eta \in \mathbb{R}}$. Using the canonical $\mathrm{CR}[E, A]=\mathrm{i}$ for the generators, the following relation holds for any $\xi$ and $\eta$

$$
\mathrm{e}^{\mathrm{i} \xi E} \mathrm{e}^{-\mathrm{i} \eta A} \mathrm{e}^{-\mathrm{i} \xi E}=\mathrm{e}^{\mathrm{i} \eta \xi} \mathrm{e}^{-\mathrm{i} \eta A}
$$

which particularizes to (5) for $\eta=1$ and $\xi=\alpha_{x}-\alpha_{x+1}$.

In a finite-dimensional Hilbert space, the role of generators loses its meaning. Nonetheless, we can define a set of unitary operators, the (discrete) Schwinger-Weyl group, that satisfy the relation (6). Notice that this entails a change of paradigm. We are abandoning an approach in terms of the algebra of generators in favor of an alternative approach in terms of its group, enabling us to explore the global features of the topology. This concept is elaborated in the Appendix. Observe that the operator $\mathrm{e}^{-\mathrm{i} \eta A}$ acts as a translation of the electric field, since $\mathrm{e}^{\mathrm{i} \eta A} E \mathrm{e}^{-\mathrm{i} \eta A}=E+\eta$. A similar role is played by the elements of the Weyl group with $\xi=0$ with respect to $A$.

Let us now consider an $n$-dimensional Hilbert space and choose an orthonormal basis $\left\{\left|v_{k}\right\rangle\right\}_{1 \leq k \leq n}$, which will be called the electric field basis, and define a unitary operator $U$ that performs a cyclic permutation of the basis states:

$$
U\left|v_{k}\right\rangle=\left|v_{k+1}\right\rangle \quad \text { for } k<n, \quad U\left|v_{n}\right\rangle=\left|v_{1}\right\rangle
$$

We will call the orthonormal eigenbasis of $U$ the vector potential basis. The operator $V$ conjugated to $U$ is diagonal in the electric field basis, with $V\left|v_{k}\right\rangle=\mathrm{e}^{-\mathrm{i} 2 \pi k / n}\left|v_{k}\right\rangle$, and it cyclically permutes the elements of the vector potential basis. Since $U^{n}=V^{n}=\mathbb{I}$, the sets $\left\{U^{k}\right\}_{1 \leq k \leq n}$ and $\left\{V^{k}\right\}_{1 \leq k \leq n}$ are unitary representations of the group $\mathbb{Z}_{n}$ of integers modulo $n$. The set of all the products between $U$ and $V$ and their integer power constitutes the Schwinger-Weyl group [29, 30, 31]. The CR between the elements of this group yield the relation

$$
V^{-k} U^{\ell} V^{k}=\mathrm{e}^{\mathrm{i} \frac{2 \pi}{n} k \ell} U^{\ell} \quad \text { with } k, \ell \in \mathbb{Z} .
$$

The multiplication law (8) satisfied by the $\mathbb{Z}_{n}$ operators is the discrete form of (6), valid for $U(1)$ operators.

Once the correspondences $U_{x, x+1} \leftrightarrow \mathrm{e}^{-\mathrm{i} A_{x, x+1}}$ and $V_{x, x+1} \leftrightarrow \mathrm{e}^{-\mathrm{i} E_{x, x+1}}$ has been set up for all links, we can construct an Abelian theory which represents an approximation to the lattice QED Hamiltonian (1) with a local $\mathbb{Z}_{n}$ invariance [32, 33]. One of the earliest examples of a pure gauge model with a $\mathbb{Z}_{n}$ invariance, used to approximate a $U(1)$ theory in the $n \rightarrow \infty$ limit, was given in [34]. The dynamics of the new model is determined by the following variant of the Hamiltonian (1)

$$
H_{n}=-t \sum_{x}\left(\psi_{x}^{\dagger} U_{x, x+1} \psi_{x+1}+\text { H.c. }\right)+m \sum_{x}(-1)^{x} \psi_{x}^{\dagger} \psi_{x}+\frac{g_{n}^{2}}{2} \sum_{x} f\left(V_{x, x+1}\right),
$$

where $f(V)$ is a suitable Hermitian operator, diagonal in the electric field basis, which represents the discretized free-field electromagnetic Hamiltonian. Unlike in the QLM, 
the ladder operators $U$ permute the electric field basis states on a circle, and transitions between neighboring states all occur with the same amplitude. These features are a consequence of the request that the minimal-coupling structure in (1) is preserved in its finite-dimensional link counterpart. The function $f(V)$ is so far arbitrary. We shall introduce a function $f(V)$ that, like $E^{2}$ in (1), has a single minimum. A simple choice is

$$
f\left(V_{x, x+1}\right)=\frac{1}{4}\left(V_{x, x+1}-\mathbb{I}\right)\left(V_{x, x+1}^{\dagger}-\mathbb{I}\right) .
$$

Since the eigenvalues of $V$ are $v_{k}=\mathrm{e}^{-\mathrm{i} 2 \pi k / n}$, the operator 10 has eigenvalues $S\left(k_{x, x+1}\right)=\left(\sin \frac{\pi k_{x, x+1}}{n}\right)^{2}$. For the low-energy states around the minimum at $k=0$, the spectrum of $f(V)$ is quadratic like the energy $E^{2}$ associated to the electric field in the original model. See Fig. 1. We remark that the choice of a function for the electric field energy does not affect the discretization of the gauge theory, as long as it is characterized by a single minimum. The energy term 10 is characterized by a nondegenerate ground state. Another interesting possibility is obtained by the replacement $V \rightarrow \mathrm{e}^{-\mathrm{i} \pi / n} V$, yielding two degenerate minima at $k=-1$ and $k=0$, which could be of interest for studying the effects of terms that break the chiral symmetry of the gauge field [35, 36, 12]. As expected, in the link discretization process the $U(1)$ gauge symmetry of the original theory becomes a $\mathbb{Z}_{n}$ symmetry. Indeed, due to condition (8), the Hamiltonian $H_{n}$ is invariant under the transformation

$$
H_{n} \rightarrow \prod_{x}\left(T_{x}^{\dagger}\right)^{\nu_{x}} H_{n} \prod_{y}\left(T_{y}\right)^{\nu_{y}}
$$

where $\nu_{x}$ is an arbitrary integer-valued function on the lattice, and

$$
T_{x}=\mathrm{e}^{\frac{2 \pi \mathrm{i}}{n}\left(\psi_{x}^{\dagger} \psi_{x}+\frac{(-1)^{x}-1}{2}\right)} V_{x, x+1} V_{x-1, x}^{\dagger}
$$

with $\left(T_{x}\right)^{n}=\mathbb{I}$, represents the discretized analogue in the Schwinger-Weyl theory of $\mathrm{e}^{\mathrm{i} G_{x}}$ [see Eq. (3)], obtained by the correspondence $\mathrm{e}^{-\mathrm{i} E} \rightarrow V$. Due to the arbitrariness of $\nu_{x}$, the gauge-invariance condition is equivalent to

$$
\left[H_{n}, T_{x}\right]=0, \quad \forall x .
$$

In the $\mathbb{Z}_{n}$ theory, the Hilbert subspace of physical states is determined by a generalized Gauss's law:

$$
\mathcal{H}_{T}=\left\{|\phi\rangle \in \mathcal{H}, T_{x}|\phi\rangle=|\phi\rangle \text { for all sites } x\right\} .
$$

In the reference basis $\left\{\left|n_{x}, v_{x, x+1}\right\rangle\right\}$, determined by the eigenvalues of $V_{x, x+1}$ and of $\psi_{x}^{\dagger} \psi_{x}$, namely the occupation numbers $n_{x} \in\{0,1\}$, the gauge invariance request translates into a condition on the eigenvalues. Since each $T_{x}$ acts nontrivially on site $x$ and the adjacent links, the physical subspace (14) is spanned by the reference basis states satisfying

$$
\mathrm{e}^{\frac{2 \pi \mathrm{i}}{n}\left(n_{x}+\frac{(-1)^{x}-1}{2}\right)} v_{x, x+1} v_{x-1, x}^{*}=1, \quad \forall x .
$$

If an even site with $(-1)^{x}=1$ is empty, the eigenvalues of $V$ in neighboring links must be equal, while if it is occupied, they will be related by $v_{x, x+1}=\mathrm{e}^{-2 \pi \mathrm{i} / n} v_{x-1, x}$. On 


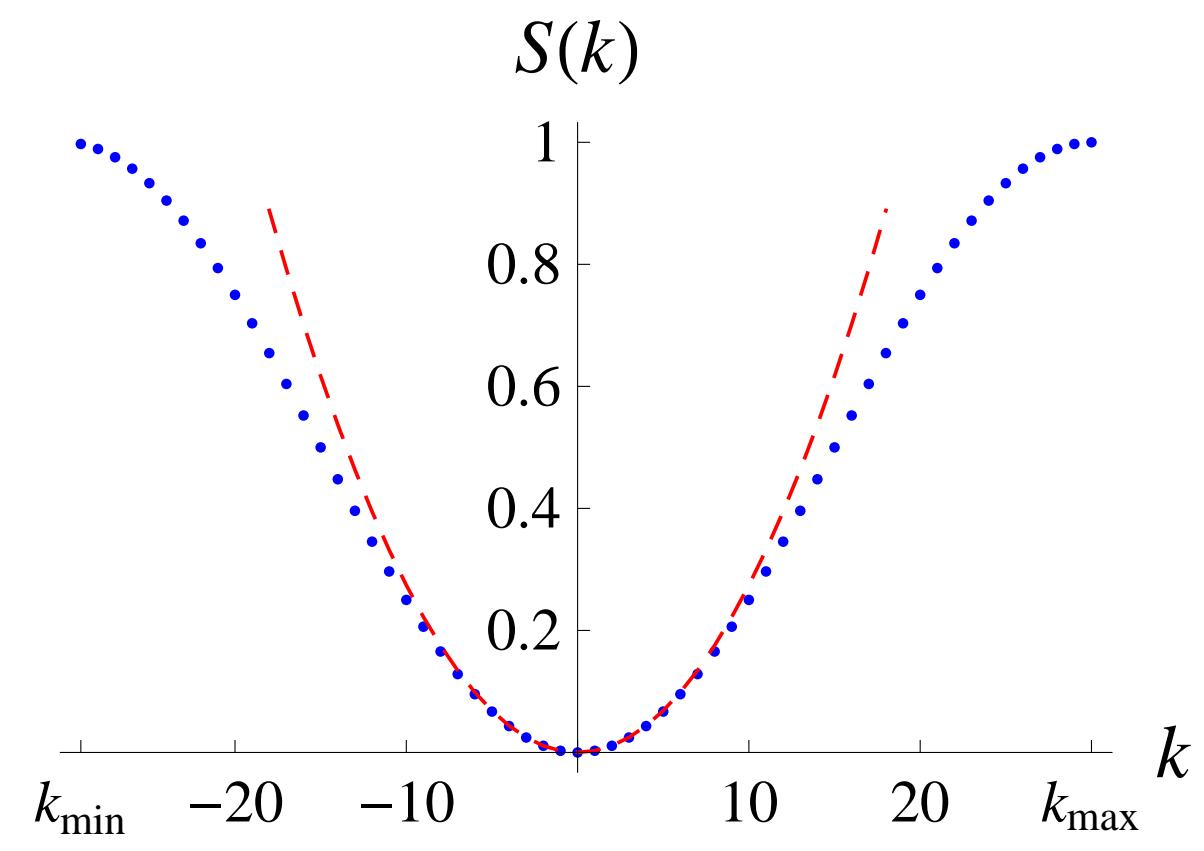

Figure 1. Spectrum $S\left(k_{x, x+1}\right)$ of the operator $f\left(V_{x, x+1}\right)$ defined in 10$)$. The dashed (red) line represents the quadratic approximation of the spectrum close to its minimum at $k=0$.

the other hand, in odd sites with $(-1)^{x}=-1$, the eigenvalues of $V$ are equal if the site is occupied, while they are related by $v_{x, x+1}=\mathrm{e}^{2 \pi \mathrm{i} / n} v_{x-1, x}$ otherwise. This is in agreement with Dirac's picture in which the absence of a particle in a negative-energy site is equivalent to the presence of an antiparticle. We can represent these situations by visualizing the eigenstates of each $V$ as $n$ points placed at an angular distance of $2 \pi / n$ on a circle. Figure 2 displays the state of an occupied even site. The action of the operator $U_{x, x+1}\left(U_{x, x+1}^{\dagger}\right)$ induces a counterclockwise (clockwise) jump of the eigenstate of $V$ to a neighboring state on the circle. The correlation between the jump in the link eigenstate and the charge displacement in the terms $\psi_{x}^{\dagger} U_{x, x+1} \psi_{x+1}$ (and conjugates) keeps the system in the physical subspace $\mathcal{H}_{T}$.

Although the approximation of Wilson's model with a $\mathbb{Z}_{n}$ lattice gauge theory improves with increasing $n$, interesting phenomenology emerges also at small link dimension: the $\mathbb{Z}_{3}$ theory is already a good test bed for the analysis of electric flux string breaking [12, 37]. Moreover, $\mathbb{Z}_{n}$ theories are relevant per se, being related to the problem of confinement in QCD [33].

Let us finally remark that an extension to higher dimensional lattices would involve a plaquette term in the Hamiltonian with a product of four $U$ matrices [25]. The $\mathbb{Z}_{n}$ theory can be generalized by a proper modification of Gauss's law, which takes into account all the terms in the divergence of the electric field. 


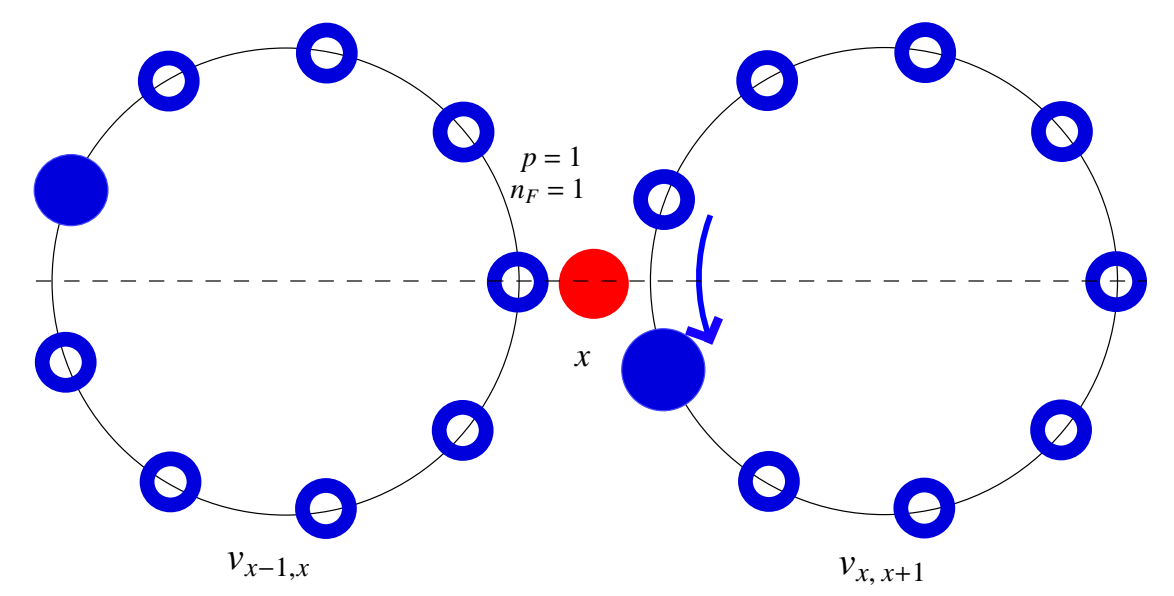

Figure 2. The state of two links (large circles) neighboring an even site [central full dot, $\left.p=(-1)^{x}=1\right]$. In this example, the site is occupied. A full dot on the circle represents the occupied eigenstate of $V$, while the empty dots are the other eigenstates. Observe that unitarity entails probability conservation: the full dot never leaves the circle under (7).

\section{Implementation strategy}

The implementation of the Hamiltonian (9) is a difficult task due to the presence of the correlated hopping terms $\psi_{x}^{\dagger} U_{x, x+1} \psi_{x+1}$, describing elementary processes in which the hopping $x \rightarrow x+1$ of a fermion to a nearest-neighboring site is always associated with an electric-field change on the link $(x, x+1)$ between the two sites. Recent results indicate that it is possible to engineer a simpler Hamiltonian, and then obtain the gauge theory by imposing the gauge-invariance constraint(s) by assigning an energy penalty to the non gauge-invariant subspace [12, 38] or by implementing Zeno constraints [21, 39, 40, 41, 42]. We shall follow the first path, and induce the correlated hopping in (9) through a secondorder effective gauge-invariant Hamiltonian.

In view of implementing a Hermitian term in the Hamiltonian, it is more convenient to express the local gauge-invariance condition $T_{x}|\phi\rangle=|\phi\rangle$ as

$$
\Gamma_{x}|\phi\rangle=0, \quad \Gamma_{x}=\left(T_{x}-1\right)\left(T_{x}^{\dagger}-1\right),
$$

so that the physical space is the kernel of the positive operator

$$
\Gamma=\sum_{x} \Gamma_{x}
$$

The dynamical conservation of the gauge condition for all sites can be enforced by adding a large term proportional to $\Gamma$ to the Hamiltonian. Notice that the constraint is diagonal in the reference basis $\left\{\left|n_{x}, v_{x, x+1}\right\rangle\right\}$.

Let us consider a Hamiltonian that involves uncorrelated hopping of fermions between nearest-neighbor sites and transitions, with equal amplitudes, between neighbor link states on a circle:

$$
H_{(0)}=-\tilde{t} \sum_{x}\left(\psi_{x}^{\dagger} \psi_{x+1}+\psi_{x+1}^{\dagger} \psi_{x}\right)-\tilde{w} \sum_{x}\left(U_{x, x+1}+U_{x, x+1}^{\dagger}\right)+H_{d}
$$


The part $H_{d}$ includes all the terms that are diagonal in the reference basis, such as the fermion mass term $m \sum_{x}(-1)^{x} \psi_{x}^{\dagger} \psi_{x}$, the gauge field energy $\left(g_{n}^{2} / 2\right) \sum_{x} f\left(V_{x, x+1}\right)$, and possibly proper counterterms.

The fermion hopping terms in (18) emerge naturally in condensed matter physics when a tight-binding approximation is assumed, in which only the lowest band of the lattice is energetically accessible. The matter field can be represented by a fermionic atomic species: the operator $\psi_{x}^{\dagger}$ creates an atom in the fundamental Wannier function centered on the lattice site $x$ [43]. Tunneling between neighboring sites provides the mechanism for the hopping processes $\psi_{x}^{\dagger} \psi_{x+1}$. The staggered structure is obtained by modulating the depth of the lattice wells [12]. A physical implementation of the action of the operators $U$ on links is less obvious, since it is first of all necessary to identify a proper system in which transitions between adjacent levels on a circle occur with the same amplitude (see Fig. 2). A possible implementation tool is represented by a longitudinal array of transverse ring-shaped lattices [44, with their axes aligned with $x$, each one representing a link and confining a single boson or fermion (the statistics being immaterial, since each ring contains one particle). The system is represented in Fig. 3. In the tight-binding regime, the fundamental Wannier states, centered on ring lattice sites, can be identified with the link reference basis states $\left|v_{x, x+1}\right\rangle$. Link particles are associated with the field operator $c_{(i) x, x+1}$, with $i=1, \ldots, n$ the site index. Hopping between neighboring sites on the link yields the processes

$$
U_{x, x+1}:=\sum_{i=1}^{n} c_{(i+1) x, x+1}^{\dagger} c_{(i) x, x+1}, \quad U_{x, x+1}^{\dagger}:=\sum_{i=1}^{n} c_{(i) x, x+1}^{\dagger} c_{(i+1) x, x+1},
$$

with $c_{(n+1)} \equiv c_{(n)}$. Transition between different rings are instead forbidden by a large energy barrier. Thus, the system and the hopping mechanism provide both the circular structure and the equality of transition amplitudes. Other possible implementations could involve a coupling between the internal levels of an atom confined on the link. However, in this case the unitary properties of transitions do not emerge by symmetry, and would require a fine tuning of the transition amplitudes [45].

While the term $H_{d}$ does not couple orthogonal eigenspaces of $\Gamma_{x}$, each nondiagonal term in (18) maps a state $|\phi\rangle$ of the physical space $\mathcal{H}_{T}=\operatorname{ker} \Gamma$ into another eigenstate $\left|\phi^{\prime}\right\rangle$ of each $\Gamma_{x}$ such that

$$
\Gamma\left|\phi^{\prime}\right\rangle=2 \gamma_{n}\left|\phi^{\prime}\right\rangle
$$

where $\gamma_{n}=2(1-\cos (2 \pi / n))$ is the first excited eigenvalue of $\Gamma_{x}$, and the factor 2 is related to the fact that each fermion hopping or Rabi transition on a link affects the eigenvalue of $\Gamma_{x}$ in two neighboring sites. The implementation strategy consists in adding to $H_{(0)}$ a term which induces a large energy cost for going out of the physical space, thus obtaining

$$
H_{(1)}=H_{(0)}+\gamma_{n}^{-1} u \Gamma .
$$

If $u$ is much larger than the parameters appearing in $H_{(0)}$, the evolution of an initial 


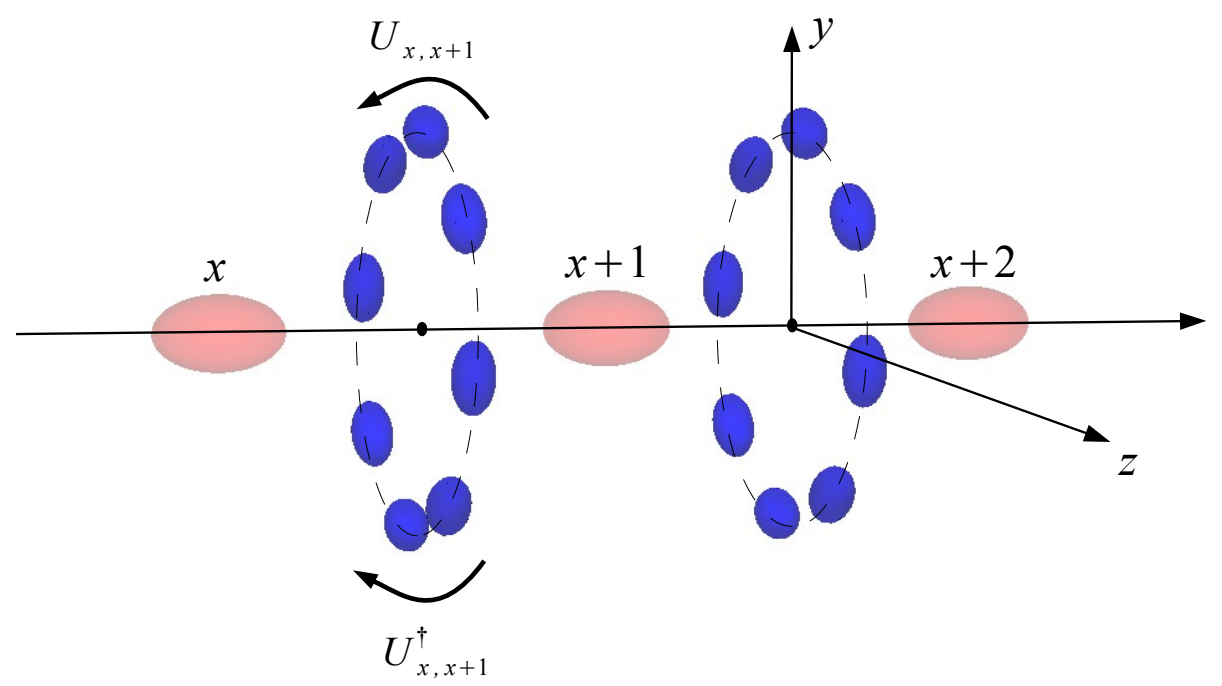

Figure 3. Scheme of the physical implementation of the model. The fermions are confined in longitudinal lattice sites [light (red) spots], while the links are represented by ring-shaped transverse lattices, each one hosting one boson or fermion. The link particle is tightly confined in the longitudinal direction, while it can hop between nearest-neighbor sites [dark (blue) spots] on each ring lattice.

state in the physical subspace $\mathcal{H}_{T}$ is approximately given by the effective Hamiltonian

$$
H_{\mathrm{eff}}=P H_{(0)} P-P H_{(0)} Q\left(\gamma_{n}^{-1} u \Gamma\right)^{-1} Q H_{(0)} P,
$$

with $P$ the projection operator on $\mathcal{H}_{T}$ and $Q=\mathbb{I}-P$. Using the fact that $P H_{(0)} P=$ $P H_{d} P$, and observing that $H_{(0)}$ couples $\mathcal{H}_{T}$ only with the eigenspace of $\Gamma$ belonging to $2 \gamma_{n}$, Eq. 222) simplifies to

$$
H_{\text {eff }}=P H_{d} P-(2 u)^{-1} P H_{(0)} Q H_{(0)} P .
$$

A straightforward computation of the second term yields, up to immaterial constants, the final result

$$
H_{\mathrm{eff}}=P\left[H_{d}-\frac{\tilde{t} \tilde{w}}{u} \sum_{x}\left(\psi_{x}^{\dagger} U_{x, x+1} \psi_{x+1}+\text { H.c. }\right)+\frac{\tilde{t}^{2}}{u} \sum_{x} n_{x}\left(1-n_{x+1}\right)\right] P,(
$$

in which the correlated hopping terms in (9) appear. The counterterms in $H_{d}$ can be tuned to cancel the undesired (though gauge-invariant) contribution in (24). Despite the necessity to couple each site with the neighboring links through $\Gamma_{x}$, this strategy can be more convenient than a direct implementation of (9), since the couplings between sites and links are all diagonal in the reference basis. It should be also noted that the operator $\Gamma$ can be replaced by any positive operator whose kernel is $\mathcal{H}_{T}$.

\section{Conclusions and outlook}

We have discussed a lattice gauge model with a discrete $\mathbb{Z}_{n}$ Abelian symmetry. The model represents an approximation to Wilson's lattice QED, that improves as the 
dimension $n$ of the link Hilbert space increases. The obstacles to an experimental implementation of the model can be overcome through the use of effective dynamics. Further research will be devoted to two main avenues. The first one is the identification of an atomic or condensed-matter system which is suitable for an experimental implementation. The second is the application of the techniques developed in this Letter to non-Abelian gauge theories. Moreover, we shall scrutinize the convergence of the $\mathbb{Z}_{n}$ models towards $U(1)$, also in comparison with the QLM, and analyze in detail the phenomenology of the model, including chiral symmetry breaking and confinement.

\section{Acknowledgements}

This work was partially supported by PRIN 2010LLKJBX on "Collective quantum phenomena: from strongly correlated systems to quantum simulators," and by the Italian National Group of Mathematical Physics (GNFM-INdAM).

\section{Appendix A. Weyl group representation in infinite dimensions}

Consider two Hermitian operators $A$ and $E$ with eigenvalue equations

$$
E|\epsilon\rangle=\epsilon|\epsilon\rangle, \quad A|\alpha\rangle=\alpha|\alpha\rangle,
$$

and satisfying the canonical commutation relations

$$
[E, A]=\mathrm{iI} .
$$

Observe that the above commutation relations make sense in an infinite-dimensional space. Two operators satisfying Heisenberg's commutation relations generate a representation of the Weyl group. We are primarily interested in the unitary operators

$$
\mathcal{U}(\eta):=\mathrm{e}^{-\mathrm{i} \eta A}, \quad \mathcal{V}(\xi):=\mathrm{e}^{-\mathrm{i} \xi E},
$$

with $\xi, \eta \in \mathbb{R}$, which act on the eigenstates (A.1) as spectral translations (in opposite directions):

$$
\mathcal{U}(\eta)|\epsilon\rangle=|\epsilon+\eta\rangle, \quad \mathcal{V}(\xi)|\alpha\rangle=|\alpha-\xi\rangle .
$$

Using the exponential form of the unitary operators (A.3), the commutation relations (A.1) and the Baker-Campbell-Haussdorf formula in the form $\mathrm{e}^{R} \mathrm{e}^{S}=\mathrm{e}^{S} \mathrm{e}^{R} \mathrm{e}^{[R, S]}$, valid if $[R, S]$ is a multiple of the identity, one obtains the property

$$
\mathcal{U}(\eta) \mathcal{V}(\xi)=\mathrm{e}^{\mathrm{i} \eta \xi} \mathcal{V}(\xi) \mathcal{U}(\eta) .
$$

The above result expresses the noncommutativity of $E$ and $A$ at the level of the Weyl group. The derivative of A.5) with respect to $\xi$ at $(\xi, \eta)=(0,1)$ yields

$$
[E, U]=U,
$$

with $U:=\mathcal{U}(1)$, which is Eq. (2) of the Letter. Notice that by taking the derivative of A.5 with respect to $\eta$ and $\xi$ at the origin one also reobtains Eq. A.2. 
In the infinite-dimensional case A.5 implies A.6. However, only A.5 can be realized in the finite-dimensional case (for discrete values of $\eta$ and $\xi$ ) by preserving the unitarity of all the operators involved. By contrast, A.6 does not admit a finite dimensional representation that preserves the properties of both $E$ and $U$. Indeed, given two operators, $U$ unitary and $E$ Hermitian, on a finite-dimensional space, the commutation relations A.6 cannot be satisfied, since they yield $U E U^{\dagger}=E-\mathbb{I}$, which contrasts with $U E U^{\dagger}$ being isospectral to $E$ with a bounded spectrum.

In the Quantum Link model of QED, which is based on the identification of the gauge degrees of freedom with spin variables $E \rightarrow S_{z}, U \rightarrow S_{+}=S_{x}+i S_{y}$, the unitary operator $U$ is replaced with a non-unitary one in order to mantain the commutation relations $[E, U]=U$ valid. We will instead choose to maintain the unitary structure of the gauge comparator $U$ in the finite-dimensional case. To accomplish this task, we shall abandon the Heisenberg's algebra relations between the operators $A$ and $E$, which cannot be realized in finite dimensions. We will focus instead on the group relations between their complex exponentials A.5, which admit a natural extension to finitedimensional spaces through the representation of the Schwinger-Weyl group.

\section{Appendix B. The discrete Schwinger-Weyl group}

Let us consider an $n$-dimensional Hilbert space $\mathcal{H}_{n}$ and choose an orthonormal basis $\left\{\left|v_{\ell}\right\rangle\right\}_{1 \leq \ell \leq n}$. It is then possible to define a unitary operator $U$ which rotates the basis states as 30]

$$
U\left|v_{\ell}\right\rangle=\left|v_{\ell+1}\right\rangle \quad \text { for } \ell<n, \quad U\left|v_{n}\right\rangle=\left|v_{1}\right\rangle .
$$

Since $U^{n}=\mathbb{I}$, the integer powers of $U$ constitute a representation of the group $\mathbb{Z}_{n}$ of integers modulo $n$. Note that the transition between the last and the first state of the basis [last line of $[$ B.1 $]$ ] is necessary to ensure the unitarity of $U$. The eigenvalue equation

$$
U\left|u_{k}\right\rangle=\mathrm{e}^{-\frac{2 \pi \mathrm{i}}{n} k}\left|u_{k}\right\rangle
$$

with $k \in\{0,1, \ldots, n\}$, is satisfied for

$$
\left|u_{k}\right\rangle=\frac{1}{\sqrt{n}} \sum_{\ell=1}^{n} \mathrm{e}^{\frac{2 \pi i}{n} k \ell}\left|v_{\ell}\right\rangle
$$

It is now possible to define an operator $V$ which rotates the eigenbasis of $U$ as

$$
V\left|u_{k}\right\rangle=\left|u_{k-1}\right\rangle \quad \text { for } k>1, \quad V\left|u_{1}\right\rangle=\left|u_{n}\right\rangle .
$$

The operator $V$ is also unitary, with $V^{n}=\mathbb{I}$. From the definition (B.4) and the form B.3 of the eigenstates of $U$, one can easily demonstrate that $\left\{\left|v_{\ell}\right\rangle\right\}_{1 \leq \ell \leq n}$ is in fact the eigenbasis of $V$, with

$$
V\left|v_{\ell}\right\rangle=\mathrm{e}^{-\frac{2 \pi \mathrm{i}}{n} \ell}\left|v_{\ell}\right\rangle
$$

The two operators $V$ and $U$ are thus called conjugated, since each one rotates the other one's eigenbasis. It is also relevant to observe that the action of $U$ and $V$ on each 
other's eigenbasis in (B.1)-(B.4) is the finite-dimensional counterpart of the spectral translations A.4. (Notice the opposite signs.) The actions of $U$ and $V$ on a state do not commute. Indeed, comparing

$$
V U\left|u_{k}\right\rangle=\mathrm{e}^{-\frac{2 \pi \mathrm{i}}{n} k} V\left|u_{k}\right\rangle=\mathrm{e}^{-\frac{2 \pi \mathrm{i}}{n} k}\left|u_{k-1}\right\rangle
$$

with

$$
U V\left|u_{k}\right\rangle=U\left|u_{k-1}\right\rangle=\mathrm{e}^{-\frac{2 \pi \mathrm{i}}{n}(k-1)}\left|u_{k-1}\right\rangle,
$$

one obtains the relation

$$
U V=\mathrm{e}^{\frac{2 \pi \mathrm{i}}{n}} V U
$$

which can be immediately generalized to all the integer powers of the operators into 30

$$
U^{\ell} V^{k}=\mathrm{e}^{\frac{2 \pi \mathrm{i}}{n} k \ell} V^{k} U^{\ell}
$$

coinciding with Eq. (8) of the Letter. This result represents the finite-dimensional generalization of A.5. The most striking difference is that the relation (B.9) is valid only for the discrete set of integers, which implies that the differentiation which leads from A.5 to A.6), is in this case meaningless. However, the procedure leading to (B.9) is successful in preserving the unitarity of the operators involved. Thus, (B.9) is a valid starting point for a gauge theory in which the local fields act on finite-dimensional Hilbert spaces. The continuum limit is recovered by introducing the Hermitian operators $A_{n}$ and $E_{n}$, such that

$$
U^{\ell}=\mathrm{e}^{-\mathrm{i} \eta_{\ell} A_{n}}, \quad V^{k}=\mathrm{e}^{-\mathrm{i} \xi_{k} E_{n}}
$$

with $\eta_{\ell}:=\ell \sqrt{2 \pi / n}$ and $\xi_{k}:=k \sqrt{2 \pi / n}$. The Hermitian operators satisfy A.2 by taking the limit $n \rightarrow \infty$, with $\eta_{\ell} \rightarrow \eta$ and $\xi_{k} \rightarrow \xi[30]$.

\section{References}

[1] Lewenstein M, Sanpera A and Ahufinger V 2012 Ultracold Atoms in Optical Lattices: Simulating Quantum Many-Body Systems (Oxford University Press, New York).

[2] Cirac J I and Zoller P 2012 Nat. Phys. 8, 264.

[3] Bloch I, Dalibard J and Nascimbène S 2012 Nat. Phys 6, 267.

[4] Blatt R. and Roos C F 2012 Nat. Phys. 8, 277.

[5] Rothe H J 1992 Lattice gauge theories (World Scientific).

[6] Montvay I and Münster G 1994 Quantum Fields on a Lattice (Cambridge University Press, Cambridge).

[7] Wilson K 1974 Phys. Rev. D 10, 2445.

[8] Kogut J B and Susskind L 1975 Phys. Rev. D 11, 395.

[9] Susskind L 1977 Phys. Rev. D 16, 3031.

[10] Kogut J B 1979 Rev. Mod. Phys. 51, 659 (1979).

[11] Kapit E and Mueller E 2011 Phys. Rev. A 83, 033625.

[12] Banerjee D, Dalmonte M, Müller M, Rico E, Stebler P, Wiese U J and Zoller P 2012 Phys. Rev. Lett. 109, 175302.

[13] Tagliacozzo L, Celi A, Orland P and Lewenstein M 2013 Nat. Commun. 4, 2615.

[14] Zohar E, Cirac J I and Reznik B 2012 Phys. Rev. Lett. 109, 125302. 
[15] Kasamatsu K, Ichinose I and Matsui T 2013 Phys. Rev. Lett. 111, 115303.

[16] Banerjee D, Bögli M, Dalmonte M, Rico E, Stebler P, Wiese U J and Zoller P 2013 Phys. Rev. Lett. 110, 125303.

[17] Tagliacozzo L, Celi A, Zamora A and Lewenstein M 2013 Ann. Phys. (Amsterdam) 330, 160.

[18] Zohar E, Cirac J I and Reznik B 2013 Phys. Rev. Lett. 110, 125304.

[19] Zohar E, Cirac J I and Reznik B 2013 Phys. Rev. A 88, 023617.

[20] Hauke P, Marcos D, Dalmonte M and Zoller P 2013 Phys. Rev. X 3, 041018.

[21] Stannigel K, Hauke P, Marcos D, Hafezi M, Diehl S, Dalmonte M and Zoller P 2014 Phys. Rev. Lett. 112, 120406.

[22] Horn D 1981 Phys. Lett. 100B, 149.

[23] Orland P and Rohrlich D 1990 Nucl. Phys. B338, 647.

[24] Chandrasekharan S and Wiese U J 1997 Nucl. Phys. B492, 455.

[25] Wiese U J 2013 Annalen der Physik 525, 777.

[26] K. Melnikov and M. Weinstein, Phys. Rev. D 62, 094504 (2000).

[27] Fradkin E 2013 Field Theories of Condensed Matter Physics (Cambridge University Press, Cambridge, England).

[28] Peskin M E and Schroeder D V 1995 An introduction to quantum field theory (Addison-Wesley Publishing Company, Reading).

[29] Weyl H 1950 The theory of groups and quantum mechanics (Courier Dover Publications).

[30] Schwinger J and Englert B G 2001 Quantum mechanics: symbolism of atomic measurements (Springer, Berlin).

[31] Varadarajan V S 1995 Lett. Math. Phys. 34, 319.

[32] Zohar E and Burrello M 2015 Phys. Rev. D 91, 054506.

[33] Zohar E, Cirac J I and Reznik B 2015 Quantum Simulations of Lattice Gauge Theories using Ultracold Atoms in Optical Lattices arXiv:1503.02312 [quant-ph].

[34] Elitzur S, Pearson R B and Shigemitsu J 1979 Phys. Rev. D 19, 3698.

[35] Coleman S, Jackiw R and Susskind L 1975 Ann. Phys. 93, 267.

[36] Coleman S 1976 Ann. Phys. 101, 239.

[37] Pichler T, Dalmonte M, Rico E, Zoller P and Montangero S 2015, arXiv:1505.04440.

[38] Zohar E and Reznik B 2011 Phys. Rev. Lett. 107, 275301.

[39] Wu L A and Lidar D A 2002 Phys. Rev. Lett. 88, 207902.

[40] Facchi P and Pascazio S (2008) J. Phys. A: Math. Theor. 41, 493001.

[41] Wu L A, Kurizki G and Brumer P 2009 Phys. Rev. Lett. 102, 080405.

[42] Raimond J M, Sayrin C, Gleyzes S, Dotsenko I, Brune M, Haroche S, Facchi P and Pascazio S 2010 Phys. Rev. Lett. 105, 213601.

[43] Bloch I, Dalibard J and Zwerger W 2008 Rev. Mod. Phys. 80, 885.

[44] Amico L, Osterloh A and Cataliotti F 2005 Phys. Rev. Lett. 95, 063201.

[45] Celi A, Massignan P, Ruseckas J, Goldman N, Spielman I B, Juzeliūnas G, and Lewenstein M 2012 Phys. Rev. Lett. 112, 043001. 